Louisiana State University

LSU Digital Commons

$1-8-2020$

\title{
A Role for Oncostatin $M$ in the Impairment of Glucose Homeostasis in Obesity
}

\author{
Irene Piquer-Garcia \\ Germans Trias i Pujol Research Institute \\ Laura Campderros \\ Universitat de Barcelona \\ Siri D. Taxerås \\ Germans Trias i Pujol Research Institute \\ Aleix Gavaldà-Navarro \\ Universitat de Barcelona \\ Rosario Pardo \\ Universitat Autònoma de Barcelona
}

See next page for additional authors

Follow this and additional works at: https://digitalcommons.Isu.edu/biosci_pubs

\section{Recommended Citation}

Piquer-Garcia, I., Campderros, L., Taxerås, S., Gavaldà-Navarro, A., Pardo, R., Vila, M., Pellitero, S., Martínez, E., Tarascó, J., Moreno, P., Villarroya, J., Cereijo, R., González, L., Reyes, M., Rodriguez-Fernández, S., VivesPi, M., Lerin, C., Elks, C., Stephens, J., Puig-Domingo, M., Villarroya, F., Villena, J., \& Sánchez-Infantes, D. (2020). A Role for Oncostatin M in the Impairment of Glucose Homeostasis in Obesity. Journal of Clinical Endocrinology and Metabolism, 105 (3) https://doi.org/10.1210/clinem/dgz090

This Article is brought to you for free and open access by the Department of Biological Sciences at LSU Digital Commons. It has been accepted for inclusion in Faculty Publications by an authorized administrator of LSU Digital Commons. For more information, please contact ir@lsu.edu. 


\section{Authors}

Irene Piquer-Garcia, Laura Campderros, Siri D. Taxerås, Aleix Gavaldà-Navarro, Rosario Pardo, María Vila, Silvia Pellitero, Eva Martínez, Jordi Tarascó, Pau Moreno, Joan Villarroya, Rubén Cereijo, Lorena González, Marjorie Reyes, Silvia Rodriguez-Fernández, Marta Vives-Pi, Carles Lerin, Carrie M. Elks, Jacqueline M.

Stephens, Manel Puig-Domingo, Francesc Villarroya, Josep A. Villena, and David Sánchez-Infantes 


\section{A Role for Oncostatin $M$ in the Impairment of Glucose Homeostasis in Obesity}

Irene Piquer-Garcia, ${ }^{1 *}$ Laura Campderros, ${ }^{2,3^{*}}$ Siri D. Taxerås, ${ }^{1}$ Aleix Gavaldà-Navarro, ${ }^{2,3}$ Rosario Pardo, ${ }^{4}$ María Vila, ${ }^{4}$ Silvia Pellitero, ${ }^{1,5}$ Eva Martínez, ${ }^{1}$ Jordi Tarascó, ${ }^{6}$ Pau Moreno, ${ }^{6}$ Joan Villarroya, ${ }^{2,7}$ Rubén Cereijo, ${ }^{2,3}$ Lorena González, ${ }^{1}$ Marjorie Reyes, ${ }^{1}$ Silvia Rodriguez-Fernández, ${ }^{8}$ Marta Vives-Pi, ${ }_{1,5}^{8,5}$ Carles Lerin, ${ }^{9}$ Carrie M. Elks, ${ }^{10}$ Jacqueline M. Stephens, ${ }^{11}$ Manel Puig-Domingo, ${ }^{1,5}$ Francesc Villarroya, ${ }^{2,3}$ Josep A. Villena, ${ }^{4,5}$ and David Sánchez-Infantes ${ }^{1,3}$

${ }^{1}$ Department of Endocrinology and Nutrition, Germans Trias i Pujol Research Institute, Barcelona, Spain; ${ }^{2}$ Department of Biochemistry and Molecular Biomedicine, and Institute of Biomedicine, University of Barcelona, Barcelona, Spain; ${ }^{3}$ BiomedicalResearch Center (Red Fisiopatología de la Obesidad y Nutrición) (CIBEROBN), ISCIII, Madrid, Spain; ${ }^{4}$ Laboratory of Metabolism and Obesity, Vall d'Hebron Institut de Recerca, Universitat Autònoma de Barcelona, Barcelona, Spain; ${ }^{5}$ BiomedicalResearch Center (Red Fisiopatología de la Diabetes y enfermedades metabólicas) (CIBERDEM), ISCIII, Madrid, Spain; ${ }^{6}$ Department of Surgery, Germans Trias i Pujol Research Institute, Barcelona, Spain; ${ }^{7}$ Infectious Diseases Unit, Hospital de la Santa Creu i Sant Pau, Barcelona, Spain; ${ }^{8}$ Immunology Section, Germans Trias i Pujol Research Institute, Barcelona, Spain; ${ }^{9}$ Endocrinology, Sant Joan de Déu Hospital, Barcelona, Spain; ${ }^{10}$ Matrix Biology Laboratory, Pennington Biomedical Research Center, Baton Rouge, Louisiana;

${ }^{11}$ Adipocyte Biology Laboratory, Pennington Biomedical Research Center, Baton Rouge, Louisiana

ORCiD numbers: 0000-0003-0950-4363 (Carles Lerin); 0000-0001-6086-7501 (David Sánchez-Infantes).

Context: Oncostatin M (OSM) plays a key role in inflammation, but its regulation and function during obesity is not fully understood.

Objective: The aim of this study was to evaluate the relationship of OSM with the inflammatory state that leads to impaired glucose homeostasis in obesity. We also assessed whether OSM immunoneutralization could revert metabolic disturbances caused by a high-fat diet (HFD) in mice.

Design: 28 patients with severe obesity were included and stratified into two groups: (1) glucose levels $<100 \mathrm{mg} / \mathrm{dL}$ and (2) glucose levels $>100 \mathrm{mg} / \mathrm{dL}$. White adipose tissue was obtained to examine OSM gene expression. Human adipocytes were used to evaluate the effect of OSM in the inflammatory response, and HFD-fed C57BL/6J mice were injected with anti-OSM antibody to evaluate its effects.

Results: OSM expression was elevated in subcutaneous and visceral fat from patients with obesity and hyperglycemia, and correlated with Glut4 mRNA levels, serum insulin, homeostatic model assessment of insulin resistance, and inflammatory markers. OSM inhibited adipogenesis and induced inflammation in human adipocytes. Finally, OSM receptor knockout mice had increased Glut4 mRNA levels in adipose tissue, and OSM immunoneutralization resulted in a reduction of glucose levels and C $\mathrm{C} 2$ expression in adipose tissue from HFD-fed mice.

Conclusions: OSM contributes to the inflammatory state during obesity and may be involved in the development of insulin resistance. (J Clin Endocrinol Metab 105: e334-e345, 2020)

Key Words: oncostatin M, obesity, insulin resistance, inflammation

ISSN Print 0021-972X ISSN Online 1945-7197 Printed in USA

(C) Endocrine Society 2019. All rights reserved. For permissions, please e-mail: journals. permissions@oup.com

Received 23 May 2019. Accepted 2 October 2019.

First Published Online 13 October 2019.

\footnotetext{
*These authors contributed equally to this work. Abbreviations: BAT, brown adipose tissue; DMEM, Dulbecco's modified Eagle's medium; GTT, glucose tolerance test; HFD, high-fat diet; HOMA-IR, homeostatic model assessment of insulin resistance; Ig, immunoglobulin; IHF, immunohistofluorescence; ITT, insulin tolerance test; LIF, leukemia inhibitory factor; MAPK, mitogen-activated protein kinas; OSM, oncostatin M; OSMR, oncostatin M receptor; SGBS, Simpson Golabi Behmel syndrome; WAT, white adipose tissue
} 
O besity has reached epidemic proportions worldwide, and its prevalence has doubled in the last 30 years. The prevalence of obesity affects more than $25 \%$ of the population, and a remarkable $60 \%$ of the population is overweight. Moreover, it has been estimated that approximately 350 million people have diabetes, representing almost $10 \%$ of the global population (1). White adipose tissue (WAT) is a crucial regulator of energy balance and glucose homeostasis owing to its function as a lipid-storing and endocrine organ. Obesity is characterized by chronic, low-grade adipose tissue inflammation accompanied by an increased presence of various proinflammatory immune cells, including macrophages (2). The first wave of macrophage accumulation in WAT during the early phase of obesity is essential for healthy expansion and remodeling of adipose tissue (3). However, chronic infiltration of adipose tissue by macrophages, their polarization from an M2 anti-inflammatory state to an M1-like inflammatory state, and increased expression and secretion of proinflammatory cytokines have been shown to negatively impact systemic glucose homeostasis (4-7). Moreover, inflammatory processes may affect the browning capacity of WAT and by this means may also contribute to obesityassociated metabolic disease. Indeed, it has been demonstrated that the immune cell infiltration of subcutaneous WAT creates a deleterious inflammatory microenvironment that impairs the capacity of precursor cells to differentiate into thermogenically active beige adipocytes (8). Understanding the crosstalk between immune cells and adipocytes in the context of obesity is essential to define the pathological bases of metabolic diseases such as insulin resistance and type 2 diabetes.

In last decade, oncostatin M (OSM) has appeared as a relevant factor in the pathophysiology of obesity and other diseases (9). Indeed, it has been reported that OSM is an important target for breast cancer and inflammatory bowel disease $(10,11)$. In support of this notion, OSM immunoneutralization in animal models can improve these diseases $(10,11)$. OSM is a gp130 cytokine with its own specific receptor, OSMR, that heterodimerizes with gp130 and mediates the majority of OSM actions (12). OSM shares substantial sequence identity with leukemia inhibitory factor (LIF) (13) and can modulate a variety of biological processes, such as liver development and regeneration $(14,15)$, hepatic insulin resistance and steatosis (16), inflammation (17), and cardiomyocyte dedifferentiation and remodeling (18). OSM has been also reported to promote bone formation and to inhibit adipogenesis (19-24).
Previously, we demonstrated that OSM was overproduced in WAT under obese conditions in mice and humans (25). In addition, we observed that OSM treatment inhibited adipogenesis in brown adipose tissue (BAT) and browning of WAT in vitro and in vivo using murine models (26). In this study, we aimed to decipher the role of OSM in promoting the inflammatory state that underlies the altered glucose homeostasis in obesity. We also addressed the therapeutic potential of OSM immunoneutralization to prevent an impairment in glucose homeostasis in vivo.

\section{Materials and Methods}

\section{Human subject characteristics}

Forty-five patients with severe obesity (body mass index $>35 \mathrm{~kg} / \mathrm{m}^{2}$ and comorbidities including type 2 diabetes, hypertension, or dyslipidemia) were included in our cohort (Table 1). Patients were stratified into two groups according to their fasting glycemia: normoglycemic patients (glucose $<100 \mathrm{mg} / \mathrm{dL})(\mathrm{n}=26)$ and hyperglycemic patients (glucose $>100 \mathrm{mg} / \mathrm{dL})(\mathrm{n}=19)$. All patients were evaluated by the same endocrinology specialist (S.P.) according to criteria for bariatric surgery formulated in the Spanish Position Statement among the Obesity, Endocrinology, Diabetes and Surgery Societies (27). Demographic and clinical data, including age, history of diabetes, and hypertension were recorded for all subjects.

\section{Serum samples and tissues}

Serum samples from the study subjects were collected after a 12-hour fasting period. All samples were stored at $-80^{\circ} \mathrm{C}$ in the Biobank of the Health Sciences Research Institute Germans Trias i Pujol Foundation.

Twenty-one of these 45 patients with obesity (10 normoglycemic patients [ 9 females/ 1 male] and 11 hyperglycemic patients [ 6 females $/ 5$ males]) were selected to analyze subcutaneous and visceral WAT (sWAT and vWAT, respectively) samples from the abdominal region. These 21 patients were chosen according to the following criteria: (1) available sWAT and/or vWAT biopsies to isolate the required amount of high-quality RNA, and (2) clinical history with at least body weight and blood glucose data available (28). WAT samples from patients with obesity were collected during bariatric surgery and immediately frozen at $-80^{\circ} \mathrm{C}$.

The Institutional Ethics Committee, in accordance with the Declaration of Helsinki, approved the study (PI16-025). All participants gave their written informed consent before collecting clinical data and samples.

\section{Human serological analysis}

Glucose and insulin levels, and lipid profiles (total cholesterol, high-density lipoprotein and low-density lipoprotein cholesterol, and triglycerides) were measured in the certified core clinical laboratory at the hospital. Leptin and high molecular weight adiponectin levels were measured using specific enzyme-linked immunosorbent assay kits (EZHL-80SK, Merck-Millipore and RYD-DHWAD0, R\&D Systems, respectively). The homeostatic model assessment of insulin 


\begin{tabular}{|c|c|c|}
\hline \multicolumn{3}{|c|}{$\begin{array}{l}\text { Table 1. Anthropometric } \\
\text { parameters of human subjects. }\end{array}$} \\
\hline & $\begin{array}{l}\text { Normo } \\
(n=26)\end{array}$ & Hyper $(n=19)$ \\
\hline & Mean \pm SD & Mean \pm SD \\
\hline Age (years) & $48 \pm 11$ & $52 \pm 10$ \\
\hline Sex (female/male) & $20 / 6$ & $14 / 5$ \\
\hline History of T2D & no & 7 T2D/12 pre-T2D \\
\hline $\begin{array}{l}\text { History of hypertension (yes/ } \\
\text { not) }\end{array}$ & $9 / 17$ & $7 / 12$ \\
\hline Weight $(\mathrm{kg})$ & $108 \pm 19$ & $117 \pm 39$ \\
\hline Height $(\mathrm{cm})$ & $163 \pm 0.1$ & $161 \pm 0.3$ \\
\hline Waist circumference $(\mathrm{cm})$ & $131 \pm 11.2$ & $146 \pm 37.3^{*}$ \\
\hline Body mass index $\left(\mathrm{kg} / \mathrm{m}^{2}\right)$ & $41 \pm 8.8$ & $43 \pm 10.8$ \\
\hline Glucose (mg/dL) & $91 \pm 5$ & $152 \pm 46^{*}$ \\
\hline Insulin (m.u.int/L) & $6.3 \pm 3.4$ & $7.7 \pm 4.0$ \\
\hline $\mathrm{HbA1c}(\%)$ & $5.3 \pm 0.4$ & $5.8 \pm 0.2^{*}$ \\
\hline HOMA-IR (\%) & $1.4 \pm 0.8$ & $2.5 \pm 1.3^{*}$ \\
\hline Triglyceride (mg/dL) & $135 \pm 41$ & $161 \pm 77$ \\
\hline LDL-cholesterol (mg/dL) & $95 \pm 27$ & $119 \pm 23^{*}$ \\
\hline HDL-cholesterol (mg/dL) & $41 \pm 11$ & $42 \pm 10$ \\
\hline Total cholesterol (mg/dL) & $163 \pm 34$ & $191 \pm 30$ * \\
\hline HMW-adiponectin (ng/mL) & $3016 \pm 1849$ & $2884 \pm 1835$ \\
\hline Leptin $(\mathrm{ng} / \mathrm{mL})$ & $54.9 \pm 25.1$ & $42.8 \pm 13.5$ \\
\hline
\end{tabular}

Data are shown as mean \pm (SD). T2D, type 2 diabetes. HbA1c, glycated hemoglobin; HOMA-IR, homeostatic model assessment-insulin resistance; low-density lipoprotein; HDL-Cho, high-density lipoprotein; HMW-adiponectin, high molecular weight adiponectin. ${ }^{*} P<.05$ Insulin ( $n=20$ normo and 10 hyper); HbA1c ( $n=22$ normo and 20 hyper); HOMA-IR ( $n=20$ normo and 10 hyper); triglycerides $(n=25$ normo and 11 hyper); LDL-cholesterol ( $n=24$ normo and 12 hyper); HDL-cholesterol ( $n=24$ normo and 12 hyper); total cholesterol $(n=25$ normo and 12 hyper); HMW-adiponectin ( $n=13$ normo and 8 hyper); leptin ( $n=13$ normo and 8 hyper).

resistance (HOMA-IR) was calculated with the following formula:

$$
\mathrm{HOMA}-\mathrm{IR}=\frac{\left[\text { Glucose } \frac{\mathrm{mg}}{\mathrm{dL}}\right] *[\text { Insulin } \mathrm{mU} / \mathrm{L}]}{405}
$$

\section{Human SGBS cell differentiation and OSM treatment}

Simpson Golabi Behmel syndrome (SGBS) human preadipocytes (29) were differentiated to adipose cells as reported previously (30). Briefly, preadipocytes were maintained with Dulbecco's modified Eagle's medium (DMEM)/ F12 supplemented with $10 \%$ heat inactivated fetal bovine serum, $32 \mu \mathrm{M}$ biotin and $16 \mu \mathrm{M}$ pantothenic acid. To induce their differentiation, cells were incubated with DMEM/F12 supplemented with $32 \mu \mathrm{M}$ biotin, $16 \mu \mathrm{M}$ pantothenic acid, $25 \mathrm{nM}$ dexamethasone, $500 \mu \mathrm{M}$ IBMX, $2 \mu \mathrm{M}$ rosiglitazone, $10 \mu \mathrm{g} / \mathrm{mL}$ transferrin, $20 \mathrm{mM}$ insulin, $100 \mathrm{nM}$ cortisol, and $0.2 \mathrm{nM} \mathrm{T} 3$ for 7 days and incubated with DMEM/F12 supplemented with $32 \mu \mathrm{M}$ biotin, $16 \mu \mathrm{M}$ panthotenic acid, $10 \mu \mathrm{g} /$ $\mathrm{mL}$ transferrin, $20 \mu \mathrm{M}$ insulin, $100 \mathrm{nM}$ cortisol and $0.2 \mathrm{nM}$ T3 for 7 additional days or until they reached more than $90 \%$ of differentiation. Where indicated, cells were treated with $1 \mathrm{mM} \mathrm{3}$ 3',5'-cyclic AMP (Sigma-Aldrich) and $1 \mathrm{nM}$ OSM (R\&D systems; reference 295-OM-CF) for 6 and 24 hours or with $1 \mu$ M CL316.243 (Cayman Chemicals) for 24 hours for acute response. Cells were treated with $1 \mathrm{nM}$ OSM ( 22 ng/ $\mathrm{mL}$ ) during all differentiation processes for chronic response studies.

\section{Gene expression analyses}

Total RNA was extracted from whole adipose tissue or cells using a column affinity-based methodology (NucleoSpin RNA II; Macherey-Nagel, Duren, Germany). Five hundred nanograms of total RNA were retrotranscribed into cDNA using random hexamer primers and multiscribe reverse transcriptase (TaqMan reverse transcription reagents, Thermo Fisher Scientific), following the manufacturer's instructions. Platinum Quantitative PCR SuperMix-UDG with ROX reagent (Themo Fisher) was used as master mix reagent and expression levels of each gene of interest were assessed with the specific TaqMan probes (Thermo Fisher) (28). Gene expression was calculated by the $\Delta \Delta \mathrm{Ct}$ method and expressed in arbitrary units.

\section{Animal studies}

Immunoneutralization study. Animals used in this study included male C57BL/6J mice fed a high-fat diet (HFD; 60\% kcal from fat, Research Diets Inc, D12492). A group of male mice fed a low-fat diet $(10 \% \mathrm{kcal}$ from fat, Research Diets Inc, D12450B) was used as a diet control $(\mathrm{n}=6)$. HFD feeding began at 8 weeks of age and extended for a 9-week period. After 7 weeks of HFD feeding, animals were randomized into 2 groups: (1) mice receiving intraperitoneal injections twice a week for a total dose of $250 \mu \mathrm{g}$ anti-OSM antibody (PA547022; Thermo Fisher Scientific, United States) over 2 weeks $(\mathrm{n}=6) ;(2)$ mice receiving intraperitoneal injections twice a week for a total dose of $250 \mu \mathrm{g}$ immunoglobulin (Ig) $G$ non-specific antibody (PA547309; Thermo Fisher Scientific, USA) over 2 weeks $(\mathrm{n}=6)(28)$. Antibody dosing was based on a previous study performed by Lapeire et al. (10).

Tissues were collected for total RNA analysis. All experimental procedures were approved by the Institutional Animal Care and Use Committee of the University of Barcelona following the principles outlined in the Declaration of Helsinki for animal experimental investigation.

Glucose and insulin tolerance tests. For the glucose tolerance test (GTT), mice were first fasted for 12 hours. Then, they were injected intraperitoneally with glucose $(2 \mathrm{~g} /$ $\mathrm{kg}$ body weight) and blood glucose was measured at $0,30,60$, 90, and 120 minutes. For insulin tolerance tests (ITTs), mice were fasted for 5 hours and then blood glucose was measured at $0,30,60,90$, and 120 minutes following an intraperitoneal injection of insulin ( $0.85 \mathrm{U} / \mathrm{kg}$ of body weight). Glucose levels were measured in blood using an ELITE glucometer (Bayer, Spain).

Rodent serological analysis. Blood was obtained by cardiac puncture and centrifuged at $2000 \mathrm{rpm}$ for 10 minutes at $4^{\circ} \mathrm{C}$ to obtain serum. Total cholesterol and triglyceride levels were quantified by using commercial kits based on the Trinder method (FAR Diagnostics, Verona, Italy). Nonesterified fatty acids were measured in serum samples with the NEFA-C kit (Wako Chemicals, Neuss, Germany). 
Insulin levels were determined with an ultrasensitive mouse insulin enzyme-linked immunosorbent assay kit (Crystal Chem, Zaandam, The Netherlands).

Osmr FKO mice. Male adipocyte-specific Osmr knockout $\left(\mathrm{Osmr}{ }^{\mathrm{FKO}} ; \mathrm{n}=6\right)$ and littermate floxed control $\left(\mathrm{Osmr} \mathrm{r}^{\mathrm{f} / \mathrm{fl}}\right.$; $\mathrm{n}=6$ ) mice on a C57BL/6J background, generated as described previously $(31,32)$, were fed a HFD $(45 \%$ calories from fat; Research Diets; D12451) for 20 weeks, beginning at 6 weeks of age. Body weights were obtained weekly. After 20 weeks of feeding, blood glucose levels were obtained after a 4-hour fast, mice were euthanized, and inguinal white adipose tissue (iWAT) was collected for gene expression analyses.

Western blotting. Fifty milligrams of frozen white adipose tissue were homogenized in $150 \mu \mathrm{L}$ of a buffer containing $50 \mathrm{mM}$ TrisHCl pH 7.4, $150 \mathrm{mM} \mathrm{NaCl}, 1.5 \mathrm{mM} \mathrm{MgCl}$, $1 \mathrm{mM}$ EDTA, $1 \% \mathrm{v} / \mathrm{v}$ Triton X-100, protease inhibitor cocktail (Roche), and phosphatase inhibitors $(2 \mathrm{mM}$ sodium orthovanadate, $1 \mathrm{mM}$ sodium pyrophosphate, $10 \mathrm{mM}$ sodium fluoride). Lysates were centrifuged at $16000 \mathrm{~g}$ at $4^{\circ} \mathrm{C}$ for 10 minutes. Protein concentration was measured using the bicinchoninic acid protein assay as specified by the manufacturer (Pierce, Thermo Fisher Scientific, Rockford, IL). Thirty micrograms of protein from each sample $(n=3)$ were resolved by sodium dodecyl sulfate-polyacrylamide $12 \%$ gels and transferred onto polyvinylidene fluoride membranes (GE Healthcare, Chicago, IL). Membranes were blocked for 1 hour at room temperature in PBS $1 \times$ with $0.1 \%$ Tween 20 and 5\% skimmed milk. Primary antibody rabbit anti-p44/42 MAPK (Erk1/2) (9102; Cell Signaling Technology, Danvers, MA) $1 / 1000$ and rabbit anti-Phospho-p44/42 MAPK (Erk1/2) (Thr202/Tyr204) (9101; Cell Signaling Technology) $1 / 1000$ were incubated overnight at $4{ }^{\circ} \mathrm{C}$ in PBS $1 \times-0.1 \%$ Tween 20-3\% milk. After incubation with HRP-labelled secondary antibodies for 1 hour at room temperature in PBS $1 \times-0.1 \%$ Tween $20-3 \%$ milk, membranes were developed with the ECL system (Millipore-Merck, Burlington, MA) and chemiluminescence was measured using the LAS-3000 Imager (Fujifilm, Tokyo, Japan).

\section{Histological analysis}

Samples from WAT and BAT were fixed for 24 hours in $4 \%$ paraformaldehyde and embedded in paraffin. Paraffin blocks were cut into $5-\mu \mathrm{m}$ sections and stained with hematoxylin and eosin (H\&E) or used for immunohistofluorescence (IHF) staining. For IHF, rehydrated tissue sections were blocked with $3 \%$ BSA for 1 hour at room temperature. Preparations were then incubated with rat anti-mouse F4/80 (ab6640, Abcam) or goat anti-mouse CD206 (sc-34577, Santa Cruz Biotechnology, Santa Cruz, CA, USA) antibodies at a concentration of $10 \mu \mathrm{g} /$ $\mathrm{mL}$ followed by AlexaFluor 488-conjugated anti-rat or antigoat IgG secondary antibodies (Thermo Fisher Scientific). Immunofluorescence signals were visualized under a fluorescence microscope (Leica). All signal counting procedures were performed by an observer blinded to the identity of samples.

\section{Statistical analysis}

Data are presented as mean \pm standard deviation $(\mathrm{SD})$ or standard error of the mean (for humans and mice, respectively).
Correlation analysis was implemented in SPSS Statistics (IBM). Normality of datasets was assessed with a KolmogorovSmirnoff test. Statistical significance was determined using a two-tailed t-test, a Mann-Whitney U-test for nonparametric data, or a one-way analysis of variance with Tukey's post hoc test for more than two groups. $P<.05$ was established as the statistical significance threshold for all analyses.

\section{Results}

OSM expression is elevated in sWAT and vWAT from hyperglycemic patients with obesity and correlates with Glut4, Ccl2, Nos2, and Pai1 mRNA levels

We previously demonstrated that OSM expression is elevated in conditions of severe obesity (25). However, the relationship between adipose tissue OSM expression and glycemia in patients with obesity has not been explored. Here, in an independent cohort of patients with severe obesity (Table 1), we analyzed whether OSM levels were correlated with altered glucose homeostasis in obesity, by comparing WAT OSM expression levels in hyperglycemic and normoglycemic patients. We observed that the mRNA levels of OSM were increased in sWAT and vWAT from hyperglycemic patients when compared with those in normoglycemic patients (Fig. 1A). We also found a decrease in Glut4 mRNA levels in WAT from patients with hyperglycemia (Fig. 1B), and an inverse correlation with OSM mRNA levels (Fig. 1C). Moreover, OSM expression correlated with transcript levels of some inflammatory molecules, such as Ccl2, Tnfa, and Col6 (a marker of fibrosis) (Fig. 1D-F), although no correlation with Nos2 and Pai1 expression was observed (Figs. $1 \mathrm{G}$ and $1 \mathrm{H}$ ).

In addition, we found direct positive correlations between OSM expression and insulin levels or HOMA-IR in sWAT but not in vWAT, and no correlation with body weight (Fig. 2), suggesting that OSM expression in sWAT is associated with reduced insulin sensitivity in obesity. After performing a linear regression analysis with all the clinical parameters and adjusting by sex and age, correlations between OSM expression and insulin or HOMA-IR were not maintained. However, body weight was an independent predictor of OSM gene expression (28).

\section{OSM inhibits differentiation and induces inflammatory markers in human adipocytes}

The effects of OSM on human adipogenesis have not been previously addressed. Therefore, human cells were treated with human recombinant OSM during differentiation (chronic exposure) or after cells were completely differentiated into mature adipocytes (acute 

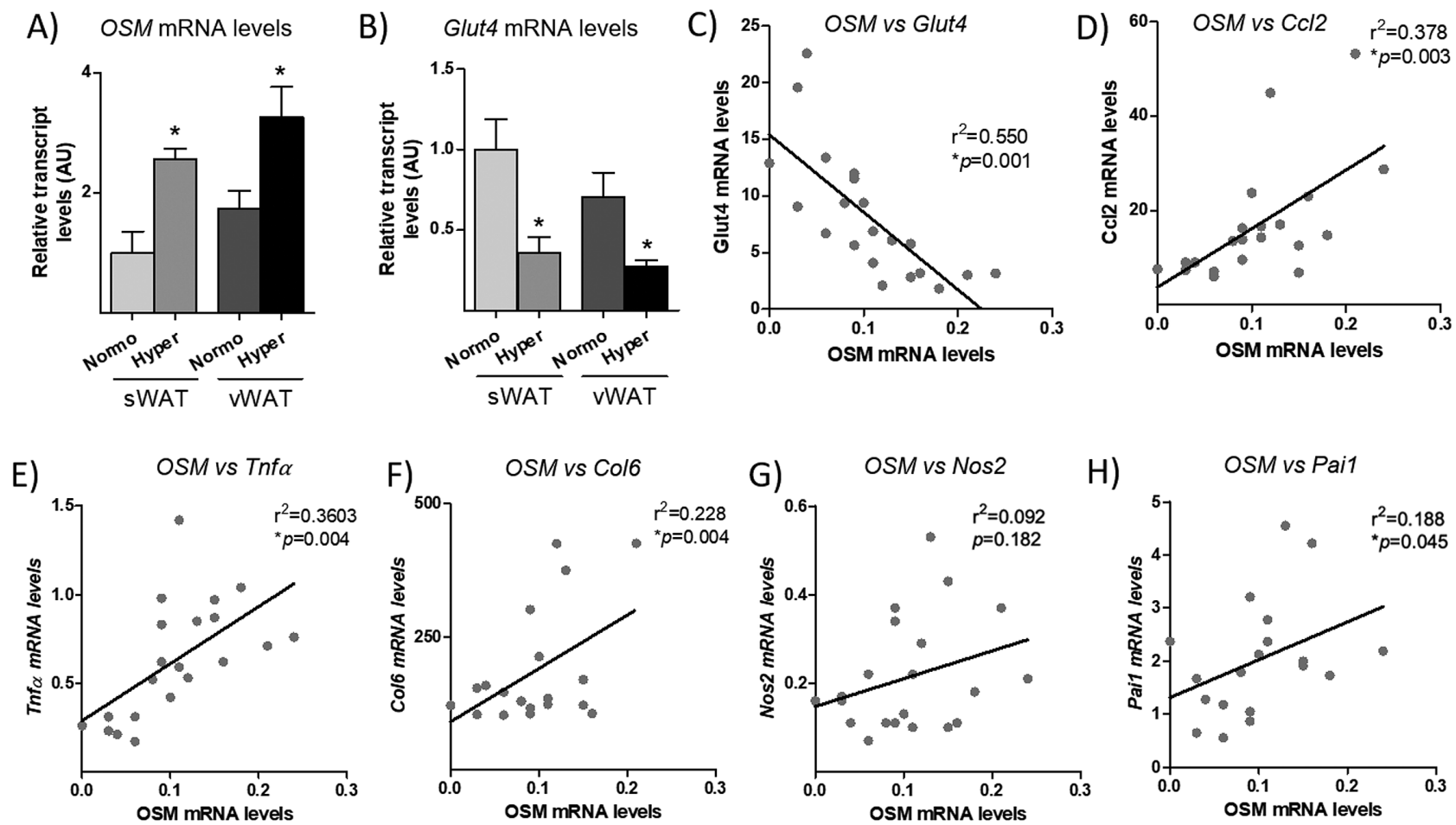

Figure 1. OSM expression is significantly elevated in sWAT and vWAT from hyperglycemic patients and correlates with Glut4, Cc/2, Tnfa and Col6 gene expression. Gene expression analysis of OSM (A) and Glut4 (B) in sWAT and vWAT from normoglycemic patients with obesity and hyperglycemic patients with obesity. Correlations between OSM expression and Glut4 (C), CCl2 (D), Tnfo (E), Col6 (F), Nos2 (G) and Pai1 (H) gene expression. Expression levels are normalized to the reference gene Ppia. Data are presented as means \pm standard error of the mean $(n=21$ samples, analytical assays performed in triplicate) $* P<.05$ vs. normoglycemic obesity group.

exposure). As shown in Fig. 3A, OSM treatment caused complete inhibition of adipogenesis. OSMR $\beta$ was expressed in both preadipocytes and mature adipocytes, with higher levels in preadipocytes (Fig. 3B). In addition, acute treatment of mature adipocytes with OSM produced significant increases in $\mathrm{Ccl} 2$, Pai1, and $\mathrm{Il}-6$ expression, and a significant decrease in Glut4 at 24 hours (Fig. 3C). Conversely, Col6 and Adipoq gene expression levels were not significantly regulated by OSM treatment (Fig. 3D).

Osmr ${ }^{\mathrm{FKO}}$ mice exhibit enhanced sWAT Glut4 mRNA levels compared with Osmr ${ }^{\mathrm{f} / \mathrm{ll}}$ controls

Since OSM was elevated in the sWAT of patients with obesity and insulin resistance, and considering that OSM treatment decreased Glut4 gene expression and increased inflammatory gene expression in human adipocytes, we next sought to evaluate the in vivo effects of diminished adipocyte OSM signaling on these parameters. To accomplish this, we used HFD-fed Osmr ${ }^{\mathrm{FKO}}$ and $O s m r^{\mathrm{fl} / \mathrm{fl}}$ control mice. Importantly, body weights did not significantly differ between genotypes (Fig. 4A). We did observe a significant increase in sWAT Glut4 mRNA levels in $O s m r^{\mathrm{FKO}}$ mice compared with floxed control mice (Fig. 4B), but transcript levels of inflammatory markers Ccl2, Nos 2 and Pai1 remained unchanged (Fig. 4C-E).
OSM immunoneutralization reduces Ccl2 expression in white and brown adipose tissue and normalizes glucose levels in diet-induced obese mice.

We aimed to evaluate the effect of systemic OSM inhibition on WAT and glucose homeostasis. To achieve this goal, we administered an anti-OSM antibody for 2 weeks to mice that had been previously fed a HFD for 7 weeks. Lower levels of phospho p44/42 mitogenactivated protein kinases (MAPKs) (p-ERK1/2), a signaling kinase activated by OSM, were observed in mice treated with anti-OSM (28), demonstrating the efficiency of the treatment of mice with the antiOSM antibody in preventing the activation of OSMinduced signaling pathway. In addition, we measured $O S M$ and OSMR $\beta$ gene expression and other pro- and anti-inflammatory markers (Tnf $\alpha$, Nos2, and Il10) and F4/80 levels by immunostaining in the eWAT of the 3 groups of mice (low-fat control diet, HFD anti-IgG, and HFD anti-OSM antibody). Significant increases in Osm, Tnfo, and Il10 mRNA levels were observed in the HFD anti-IgG group, in addition to more F4/80 positive cells, while these increases were not significant in mice treated with anti-OSM antibody. These results demonstrate the partial benefit of anti-OSM antibody injection, which was sufficient to ameliorate general inflammatory response (28). 
SWAT

A)

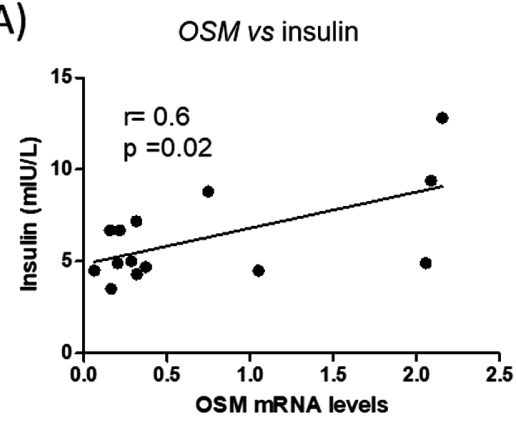

vWAT
B)

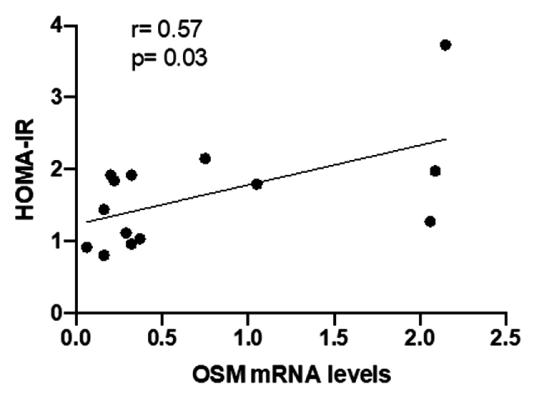

C)
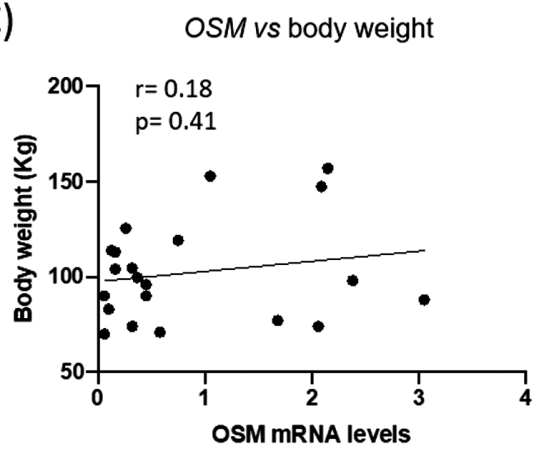

D)

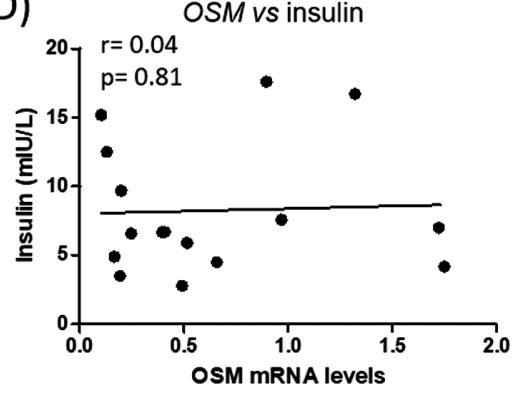

E)

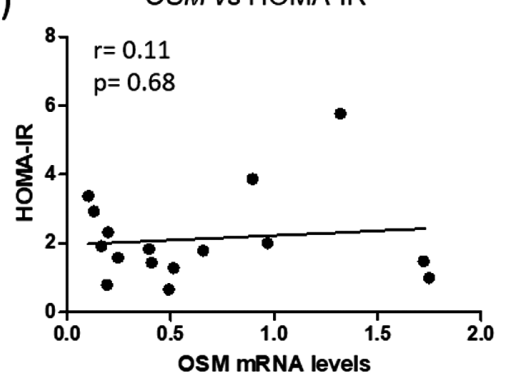

F)

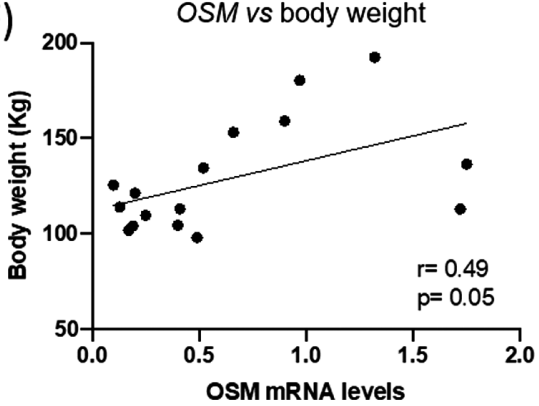

Figure 2. OSM gene expression in SWAT but not in VWAT correlates with circulating insulin levels and HOMA-IR. Correlations between OSM expression in sWAT and vWAT and insulin levels ( $A$ [ $n=14], D[n=15]), \operatorname{HOMA-IR}(B[n=12], E[n=15])$ and body weight $(C[n=21], F[n=16])$. Expression levels are normalized to the reference gene Ppia. Data are presented in arbitrary units as means \pm standard error of the mean (analytical assays performed in triplicate).

Mice treated with the anti-OSM antibody exhibited lower glucose levels compared to the anti-IgG control group, in a similar extent to those present in mice fed a low-fat control diet (Fig. 5A). Insulin levels showed a tendency to be higher in the anti-OSM antibody group compared with anti-IgG group and were significantly different from those observed in the low-fat control diet group (Fig. 5B). Consistent with our in vitro data in human adipocytes, we observed that administration of an anti-OSM neutralizing antibody lowered $\mathrm{Ccl} 2$ gene expression in eWAT and BAT of HFD-fed mice when compared with HFD-fed mice given the anti-IgG control antibody (Figs. 5D and 5E). There were no differences in $\mathrm{Ccl} 2$ expression in iWAT (Fig. 5F). PAI1 expression increased in BAT with HFD and was normalized after OSM immunoneutralization (Fig. 5G), and no changes were found in WAT (Figs. 5H and 5I). Moreover, we did not observe changes in Glut4 mRNA levels in iWAT from the 3 groups of study (Fig. 5C). As expected, compared with mice fed a low-fat control diet, HFDfed mice exhibited impaired glucose homeostasis (28) in close association with the development of obesity. Despite changes in basal glucose levels, treatment with anti-OSM antibody for 2 weeks did not induce any significant changes in glucose tolerance or insulin sensitivity compared with mice treated with anti-IgG antibody (28). Similarly, no differences in body weight or food intake were observed between IgG or anti-OSM antibody-treated mice (28). WAT and BAT weights were similar in both groups of HFD-fed mice, independently of treatment (28). Moreover, no differences in Ppar $\gamma$ expression were observed in WAT and BAT from either group on HFD (28), suggesting that adipogenesis was not affected by the neutralizing antibody. Consistent with this, adipocyte size in iWAT and eWAT depots was not affected by the treatments (data not shown).

\section{Discussion}

In this study, we report for the first time that OSM is differentially expressed in adipose tissue from hyperglycemic patients with obesity when compared with normoglycemic patients with a similar degree of obesity. In addition, we demonstrate that OSM inhibits human adipogenesis, reduces Glut4 expression, and induces an inflammatory state in human adipocytes. The inhibitory effect of OSM on adipocyte differentiation has been previously reported in different conditions (19-24). Here, 


\section{A) Adipogenesis}
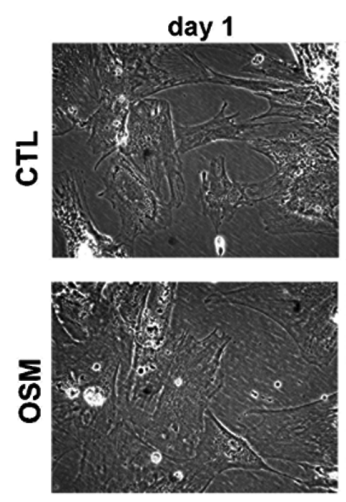
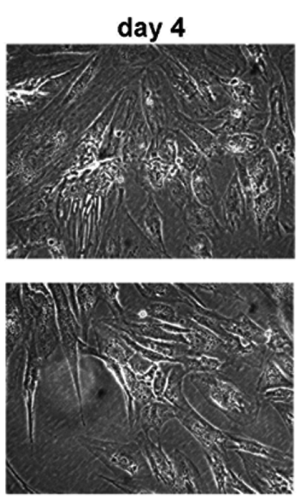
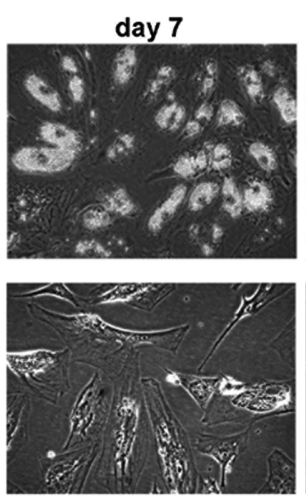

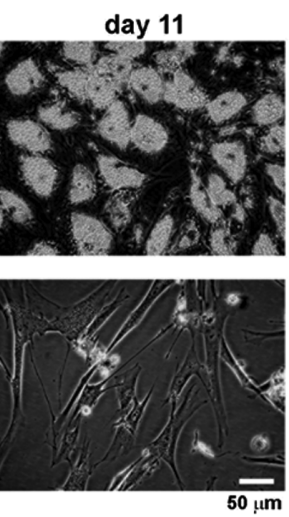

B) OSMRß mRNA levels

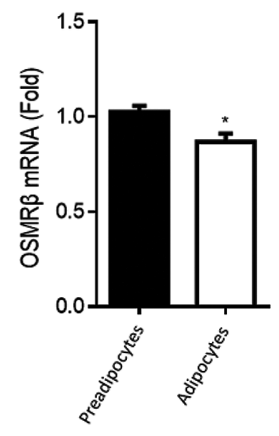

\section{C) Mature adipocytes}
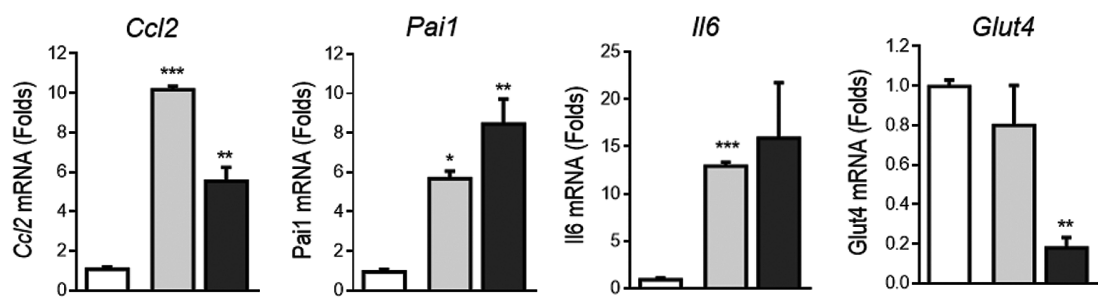

D)

Figure 3. OSM inhibits human adipogenesis and induces a proinflammatory gene expression profile in mature human adipocytes. (A) Representative optical microscopy images from human preadipocytes treated with 1 nM OSM since day 1 of differentiation. ${ }^{\star} P<0.05$ relative to control. (B) Relative mRNA levels of OSMR $\beta$ in preadipocytes and mature adipocytes. (C) Relative mRNA levels of Ccl2, Pai1, II-6 and Glut4 in human mature adipocytes treated with 1nM OSM for 6 or 24 hours. (D) Relative mRNA levels of Col6 and Adipoq. Expression levels are normalized to the reference gene Ppia. The bars represent means \pm standard error of the mean. Each figure represents an experiment independently performed 3 times with 3 replicates per sample. The mass unit concentration of $1 \mathrm{nM}$ recombinant human OSM is $\sim 22 \mathrm{ng} / \mathrm{mL}$.

we link the capacity of OSM to impair adipogenesis with its potential role to promote an inflammatory state, contributing to the development of insulin resistance in obesity. Consistent with these observations, our data in animal models also demonstrate that $\mathrm{Osmr}{ }^{\mathrm{FKO}}$ mice show higher levels of Glut4 than floxed controls, and that OSM immunoneutralization reduces inflammatory markers and decreases basal glucose levels in obese mice.

In the last decade, the enigmatic concept of "healthy obesity" has emerged in the field of metabolism (33). Whether healthy obesity is only a transient state in the progression towards metabolic disease is controversial and still a subject of intense debate. But, it is clear that some patients maintain normal glucose homeostasis in the presence of obesity (34). Metabolically healthy patients with obesity are characterized by the absence of metabolic complications such as dyslipidemia, insulin resistance, and unfavorable inflammatory profiles (35). Whereas dietary composition and physical activity are factors that have been related to metabolic health in patients with obesity, numerous studies have provided evidence implicating chronic inflammation, particularly in adipose tissue, as a key factor in determining an unfavorable metabolic outcome in patients. Indeed, higher levels of proinflammatory cytokines, including C-reactive protein, interleukin (Il)-6, Plasminogen activator inhibitor 1 (Pai1) or tumor necrosis factor alpha ( TNF $\alpha)$, have been reported in unhealthy obesity than in healthy obesity $(36,37)$.

In this regard, OSM, a cytokine from the Il-6 family, is produced by adipose tissue and highly expressed in WAT of mouse models of diet-induced and genetic obesity, as well as in humans with obesity (25). Our data showing that increased expression of OSM in WAT correlates with markers of metabolic disease, including decreased Glut4 expression, hyperglycemia, hyperinsulinemia, and increased HOMA-IR in patients with obesity, suggests that OSM is a marker of unhealthy obesity.

The correlation of OSM levels in sWAT with glycemia, but not with body weight, in patients may seem somewhat paradoxical given the finding that OSM negatively acts on precursor cells to inhibit adipocyte differentiation in vitro. A regulatory role of OSM on 
Body weight

A)

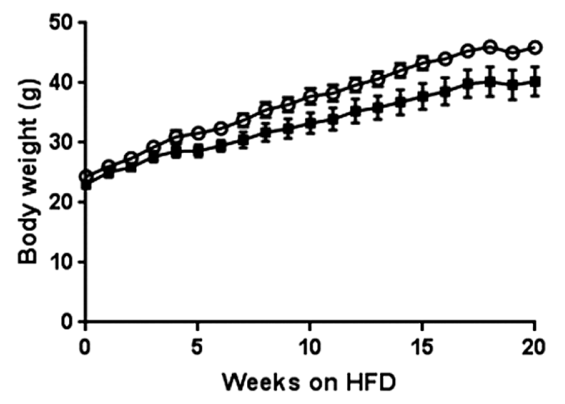

Glut4

B)

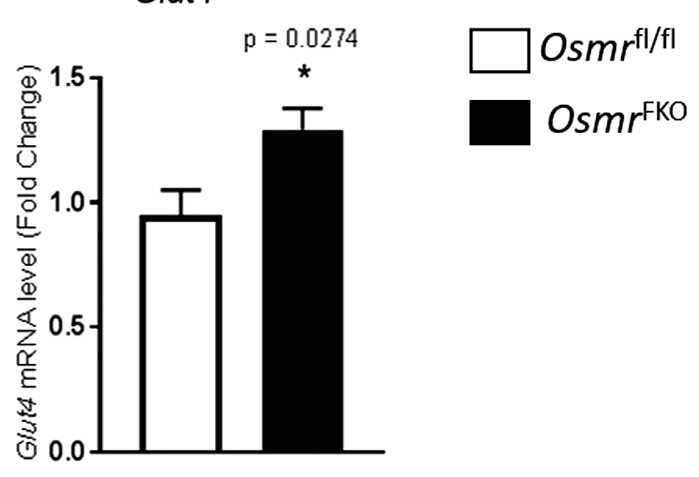

Ccl2

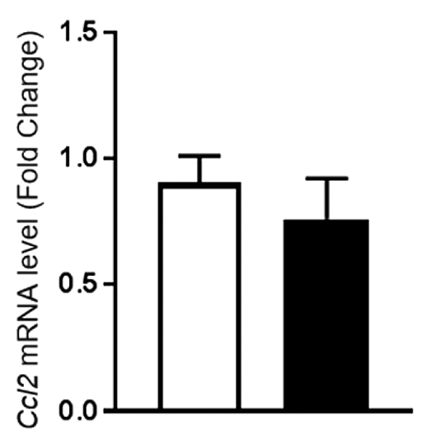

E)

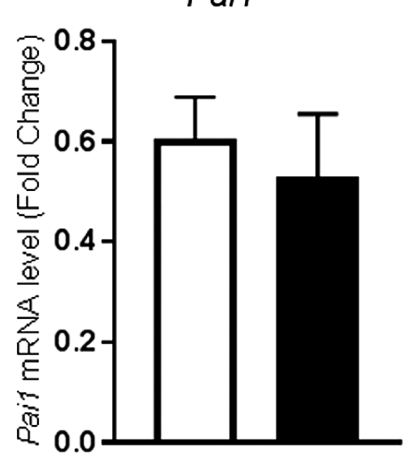

Figure 4. Osmr ${ }^{\mathrm{FKO}}$ mice exhibit elevated Glut4 mRNA levels, but no changes in inflammatory markers, compared to floxed controls. Body weight of Osmr ${ }^{\mathrm{FKO}}$ mice and control littermates fed a HFD (A). Relative mRNA levels of Glut4 (B), Nos2 (C), CCl2 (D) and Pai1 (E) in iWAT from HFD-fed $\mathrm{Osmr} r^{\mathrm{FKO}}$ mice and $\mathrm{Osmr} r^{\mathrm{fl} / \mathrm{fl}}$ controls. Data are presented in arbitrary units as means \pm standard error of the mean $(\mathrm{n}=6$ mice per group and analytical assays performed in triplicate). Expression levels are normalized to the reference gene Ppia. ${ }^{*} P<0.05$ Osmr ${ }^{F K O}$ vs. Osmr ${ }^{f l / f l}$ control mice.

adipose tissue mass is supported by studies in mice lacking whole-body OSM receptor (OSMR $\beta$ ), which show increased fat mass $(38,39)$. However, in humans, the causal factors underlying fat accretion in obesity are yet to be fully determined, and genetic or lifestyle factors (hypercaloric diets, sedentarism) may override the protective effect of OSM. On the other hand, the lack of any effect on body weight and adipose tissue mass in mice treated with an anti-OSM neutralizing antibody could be explained by (1) the short period of treatment (only 2 weeks), which was likely not sufficient to observe the effects of impaired adipocyte differentiation on WAT mass, or (2) the dosage used.

A major finding of our study is the observation of direct correlations between OSM and inflammatory markers such as the monocyte chemoattractant, $\mathrm{Ccl}$, in WAT of patients. These results are in accordance with our previous data and with studies by other researchers that reported a proinflammatory role for $\operatorname{OSM}(10,11$, 25). Moreover, the effect of OSM in inducing these inflammatory genes in human adipocytes strongly suggests a potential role for OSM in adipose tissue inflammation in patients.
In addition, OSM downregulates Glut4 in WAT as demonstrated by the decreased WAT Glut4 expression in patients with obesity and hyperglycemia, the inverse correlation with OSM expression, and the response to direct OSM exposure in human adipocytes. These results are supported by the elevation of sWAT Glut4 expression in $\mathrm{Osmr}{ }^{\mathrm{FKO}}$ mice compared with floxed controls. These data suggest a potential contribution of this cytokine to impaired insulin sensitivity. This elevation in Glut4 is unique to sWAT; as no differences were previously observed in vWAT Glut4 expression in Osmr ${ }^{\mathrm{FKO}}$ mice (31). This depot-specific expression pattern provides additional support for the possible protective role of sWAT, especially considering the increased vWAT inflammation observed in $\mathrm{Osmr} r^{\mathrm{FKO}}$ mice on both HFD and chow diets $(31,32)$.

Work by Elks et al. (32) using C57BL/6J mice demonstrated that OSM is primarily expressed in adipose tissue immune cells, and that OSMR is also expressed in cellular components of the adipose tissue immune system. Interestingly, however, Osmr expression increases only in adipocytes from HFD-fed mice (32). Further work using the adipocyte-specific $O s m r^{\mathrm{FKO}}$ 
A)

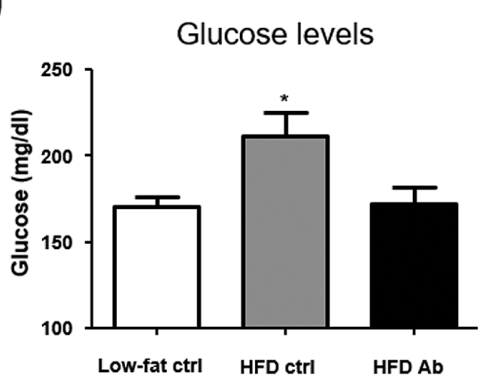

D)

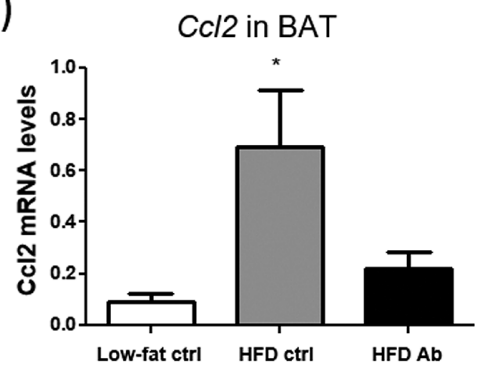

G)

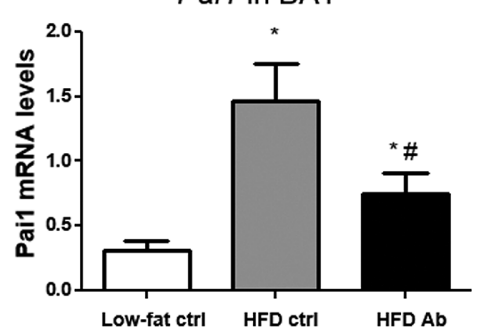

B)

Insulin levels

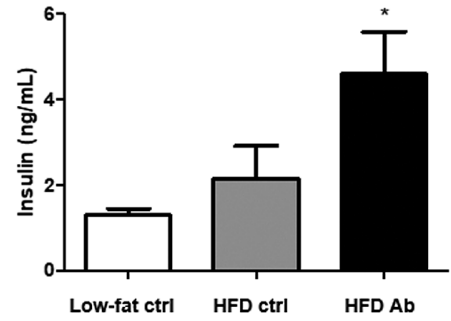

E)

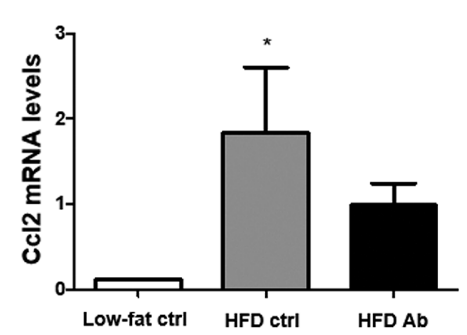

H)

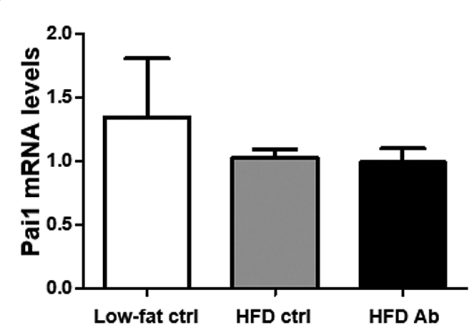

C) Glut4 in iWAT

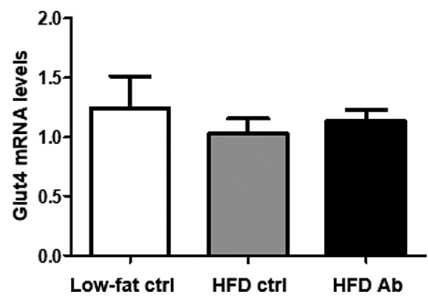

F)

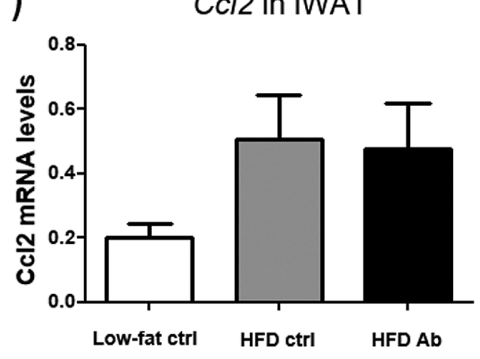

I) Pai1 in iWAT

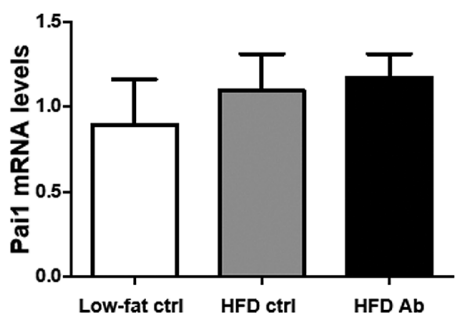

Figure 5. OSM immunoneutralization decreases C $/ 2$ expression in WAT and BAT and normalizes glucose levels. Glucose (A) and insulin (B) levels in random fed animals. Glut4 mRNA levels in iWAT (C). Relative mRNA levels of $C C l 2(D, E, F)$ and Pai1 (G, H, I) in WAT and BAT. The 3 groups correspond to mice fed low-fat control diet (control diet), mice fed a HFD and treated with unspecific lgG (HFD control), and mice fed a HFD and treated with anti-OSM Ab (HFD Ab). Data are presented in arbitrary units as means \pm standard error of the mean $(n=6$ mice per group and analytical assays performed in triplicate). ${ }^{*} P<.05$ vs control diet.

mouse model showed significant increases in adipose tissue $\mathrm{Osm}$ expression in $\mathrm{Osmr} r^{\mathrm{FKO}}$ mice compared with $\mathrm{Osmr} r^{\mathrm{f} / \mathrm{l}}$ controls, along with increased adipose tissue inflammation. These findings suggest that since OSM could not bind OSMR on Osmr $r^{F K O}$ adipocytes, it was instead acting on other cell types within adipose tissue to promote inflammation and insulin resistance $(31,32)$.

Taking into account studies indicating that adipocyte OSMR $\beta$ signaling is important for adipose tissue homeostasis $(31,32)$, we examined the effects of OSM immunoneutralization on metabolic parameters in dietinduced obese mice. Regarding the inflammatory state, we observed a significant increase in OSM, TNF $\alpha$, and Il10 mRNA levels in HFD-fed mice treated with IgG antibody, while this increase was not significant in HFDfed mice treated with anti-OSM antibody compared with mice fed a low-fat diet. The elevated levels of Il1O in HFD-fed mice might be due to a compensatory mechanism against the increasing inflammatory process.

Moreover, anti-OSM treatment reduced the HFDinduced increase in $\mathrm{Ccl} 2$ expression in vWAT and BAT, and also decreased Pai1 expression in BAT. Further, glucose levels were normalized in HFD-fed mice after the OSM immunoneutralization, although treatment with the anti-OSM antibody did not significantly alter whole body glucose tolerance or systemic insulin sensitivity. Since we did not observe changes in Glut4 expression, the mechanisms by which OSM neutralization normalized glucose levels in HFDinduced obese mice are still unclear. They may involve a reduction in the local adipose tissue inflammatory state and immune cell infiltration, as evidenced by reduction in transcript levels of chemoattractant and proinflammatory molecules, with a consequent improvement in adipocyte insulin sensitivity. However, 
the contribution of other tissues in response to treatment with this antibody cannot be disregarded. For example, the observed increase in insulin levels in anti-OSM treated mice could suggest direct effects on pancreatic $\beta$-cells. Moreover, a decrease in hepatic gluconeogenesis is also compatible with our observations. In this regard, OSM released by Kupffer cells in response to prostaglandin E2 has been shown to inhibit hepatic insulin signaling and to promote abnormal lipid accumulation (16).

Similar to studies by Komori et al. (38-40), we are confident that OSM is secreted from nonadipocyte cells in white adipose tissue under obese conditions in mice and humans, and that the expression of the OSM receptor, $O S M R \beta$, is elevated in adipose tissue in conditions of obesity. However, a clear divergence between our observations and those reported in by Komori includes the primary function of OSM signaling in adipose tissue. Our results demonstrate that OSM has proinflammatory roles on adipocytes, including induction of Ccl2 and Pai-1, and that levels of OSM in adipose tissue inversely correlated with glucose homeostasis parameters. Our observations supporting that elevated OSM levels in adipose tissue have negative effects, as part of an overall proinflammatory enhancement, are consistent with several studies by other laboratories. Lapeire et al. (10) demonstrated recently that, of all the cytokines secreted by mammary adipose tissue, OSM is the most relevant secreted factor in stimulating breast cancer progression. Also, a study by Albiero et al. (41) suggests that OSM antagonism is a strategy to restore bone marrow function in diabetes to combat the effects of elevated OSM levels produced in bone marrow macrophages in diabetics. We and others observed proinflammatory actions of OSM in adipocytes in mouse and human adipocytes $(25,42,43)$. The fact that OSM is a strong inhibitor of adipogenesis (19-24) is consistent with our proposal of OSM as a damaging factor on glucose homeostasis, as factors that limit fat cell expansion are commonly associated with metabolic dysfunction (7).

It is also important to point out that, for their studies in vivo, Komori et al. (40) used a dose of recombinant OSM to treat mice $(12.5 \mathrm{ng} / \mathrm{g}$ body weight, administered twice daily) which is at least 3 to 4 orders of magnitude above the highest reported levels of circulating OSM in humans (42). A potential explanation for the differing conclusions could be related to the use of whole-body, constitutive, Osmr knockout mice by Komori et al. Osm has well-known critical roles in liver development and hematopoiesis (14-18) and is expressed in many tissues during both embryonic development and adulthood in mice (42). OSMR $\beta$-null mice had mature-onset obesity and insulin resistance (38), but this may be the result of multiple damaging events on early development, differentiation and cell programming beyond the actual role of OSM in adipose tissue when obesity develops in adulthood.

Since immunotherapy is being used to treat several inflammatory diseases (44), the practical implication of this study is to open the window for potential approaches targeting immunoregulation of adipose tissue in obesity to prevent and treat insulin resistance.

Regardless, our study presents some limitations, including the relatively low number of human adipose tissue samples and the lack of circulating OSM levels in these patients. Moreover, a polyclonal goat IgG might be immunogenic in mice and could act by neutralizing antibody responses over a 2 -week treatment period leading to a lack of effect due to poor drug pharmacokinetics. Further studies evaluating the effect of antibody treatment at earlier timepoints would be necessary to decipher whether OSM immunoneutralization could be a potential strategy to treat insulin resistance.

In conclusion, OSM is elevated in adipose tissue from patients with obesity and impaired glucose metabolism, and inversely correlates with Glut4 expression in this tissue. Moreover, OSM promotes an inflammatory state during obesity, likely in part through the inhibition of adipogenesis, a direct reduction of the mRNA levels of Glut4 and induction of the expression of inflammatory markers. Finally, Osmr ${ }^{\mathrm{FKO}}$ mice showed elevated levels of Glut4, and OSM immunoneutralization reduced adipose tissue Ccl2 and Pail expression in obese mice, and normalized glucose levels, demonstrating the negative action of OSM on glucose homeostasis. Further studies will be necessary to decipher whether blockage of OSM signaling can be a therapeutic target in preventing the development of type 2 diabetes.

\section{Acknowledgments}

Samples from patients included in this study were provided by the IGTP HUGTP Biobank integrated in the Spanish National Biobanks Network of Instituto de Salud Carlos III (PT13/0010/0009) and they were processed following standard operating procedures with the appropriate approval of the Ethical and Scientific Committees.

Financial Support: DS-I is Investigator of the Miguel Servet Fund from Carlos III National Institute of Health, Spain. This study was supported by grants CP15/00106 and FIS17/01455 from Instituto de Salud Carlos III and the Fondo Europeo de Desarrollo Regional (FEDER) to D.S.I., grants SAF201239484 and BFU2015-64462-R, and grant SAF2017-85722R fom the Ministerio de Economía y Competitividad (MINECO/ FEDER) to J.A.V and to FV, respectively. C.M.E is supported 
by grant number K01 DK106307 from the National Institutes of Health.

\section{Additional Information}

Correspondence and Reprint Requests: David SánchezInfantes, PhD, Obesity and Type 2 Diabetes: Adipose Tissue Biology Group Leader, Germans Trias i Pujol Research Institute (IGTP), Campus Can Ruti, Carretera de Can Ruti, Camí de les Escoles s/n 08916 Badalona, Barcelona, Spain. E-mail: dsanchez@igtp.cat.

Disclosure Summary: I certify that neither I nor my co-authors have a conflict of interest as described above that is relevant to the subject matter or materials included in this Work.

Data availability: All data generated or analyzed during this study are included in this published article or in the data repositories listed in References.

\section{References}

1. WHO. Obesity and Overweight. World Health Organization; 2017. https://www.who.int/news-room/fact-sheets/detail/obesityand-overweight

2. Apostolopoulos V, de Courten MP, Stojanovska L, Blatch GL, Tangalakis K, de Courten B. The complex immunological and inflammatory network of adipose tissue in obesity. Mol Nutr Food Res. 2016;60(1):43-57.

3. Pellegrinelli V, Carobbio S, Vidal-Puig A. Adipose tissue plasticity: how fat depots respond differently to pathophysiological cues. Diabetologia. 2016;59(6):1075-1088.

4. Weisberg SP, McCann D, Desai M, Rosenbaum M, Leibel RL, Ferrante AW Jr. Obesity is associated with macrophage accumulation in adipose tissue. J Clin Invest. 2003;112(12):1796-1808.

5. Patsouris D, Li PP, Thapar D, Chapman J, Olefsky JM, Neels JG. Ablation of CD11c-positive cells normalizes insulin sensitivity in obese insulin resistant animals. Cell Metab. 2008;8(4):301-309.

6. Masoodi M, Kuda O, Rossmeisl M, Flachs P, Kopecky J. Lipid signaling in adipose tissue: connecting inflammation \& metabolism. Biochim Biophys Acta. 2015;1851(4):503-518.

7. Virtue S, Vidal-Puig A. Adipose tissue expandability, lipotoxicity and the Metabolic Syndrome-an allostatic perspective. Biochim Biophys Acta. 2010;1801(3):338-349.

8. Villarroya F, Cereijo R, Gavaldà-Navarro A, Villarroya J, Giralt M. Inflammation of brown/beige adipose tissues in obesity and metabolic disease. J Intern Med. 2018;284(5):492-504.

9. Stephens JM, Elks CM. Oncostatin M: potential Implications for Malignancy and Metabolism. Curr Pharm Des. 2017;23(25):3645-3657.

10. Lapeire L, Hendrix A, Lambein K, et al. Cancer-associated adipose tissue promotes breast cancer progression by paracrine oncostatin $\mathrm{M}$ and Jak/STAT3 signaling. Cancer Res. 2014;74(23):6806-6819.

11. West NR, Hegazy AN, Owens BMJ, et al; Oxford IBD Cohort Investigators. Oncostatin $\mathrm{M}$ drives intestinal inflammation and predicts response to tumor necrosis factor-neutralizing therapy in patients with inflammatory bowel disease. Nat Med. 2017;23(5):579-589.

12. Mosley B, De Imus C, Friend D, et al. Dual oncostatin M (OSM) receptors. Cloning and characterization of an alternative signaling subunit conferring OSM-specific receptor activation. $J$ Biol Chem. 1996;271(51):32635-32643.
13. Rose TM, Lagrou MJ, Fransson I, et al. The genes for oncostatin $\mathrm{M}$ (OSM) and leukemia inhibitory factor (LIF) are tightly linked on human chromosome 22. Genomics. 1993;17(1):136-140.

14. Kamiya A, Kinoshita T, Ito Y, et al. Fetal liver development requires a paracrine action of oncostatin $M$ through the gp130 signal transducer. Embo J. 1999;18(8):2127-2136.

15. Nakamura K, Nonaka H, Saito H, Tanaka M, Miyajima A. Hepatocyte proliferation and tissue remodeling is impaired after liver injury in oncostatin $\mathrm{M}$ receptor knockout mice. Hepatology. 2004;39(3):635-644.

16. Henkel J, Gärtner D, Dorn C, et al. Oncostatin M produced in Kupffer cells in response to PGE2: possible contributor to hepatic insulin resistance and steatosis. Lab Invest. 2011;91(7):1107-1117.

17. Wallace PM, MacMaster JF, Rouleau KA, et al. Regulation of inflammatory responses by oncostatin M. J Immunol. 1999;162(9):5547-5555.

18. Kubin T, Pöling J, Kostin S, et al. Oncostatin M is a major mediator of cardiomyocyte dedifferentiation and remodeling. Cell Stem Cell. 2011;9(5):420-432.

19. Walker EC, McGregor NE, Poulton IJ, et al. Oncostatin M promotes bone formation independently of resorption when signaling through leukemia inhibitory factor receptor in mice. J Clin Invest. 2010;120:582-592.

20. Guihard P, Danger Y, Brounais B, et al. Induction of osteogenesis in mesenchymal stem cells by activated monocytes/ macrophages depends on oncostatin M signaling. Stem Cells. 2012;30:762-772.

21. Sato F, Miyaoka Y, Miyajima A, Tanaka M. Oncostatin M maintains the hematopoietic microenvironment in the bone marrow by modulating adipogenesis and osteogenesis. PLOS ONE. 2014;9(12):e116209.

22. Song HY, Jeon ES, Kim JI, Jung JS, Kim JH. Oncostatin M promotes osteogenesis and suppresses adipogenic differentiation of human adipose tissue-derived mesenchymal stem cells. J Cell Biochem. 2007;101(5):1238-1251.

23. Smyth DC, Takenaka S, Yeung C, Richards CD. Oncostatin M regulates osteogenic differentiation of murine adipose-derived mesenchymal progenitor cells through a PKCdelta-dependent mechanism. Cell Tissue Res. 2015;360(2):309-319.

24. Song HY, Kim MR, Lee MJ, et al. Oncostatin M decreases adiponectin expression and induces dedifferentiation of adipocytes by JAK3- and MEK-dependent pathways. Int J Biochem Cell Biol. 2007;39(2):439-449.

25. Sanchez-Infantes D, White UA, Elks CM, et al. Oncostatin $\mathrm{m}$ is produced in adipose tissue and is regulated in conditions of obesity and type 2 diabetes. J Clin Endocrinol Metab. 2014;99(2):E217-E225.

26. Sánchez-Infantes D, Cereijo R, Peyrou M, Piquer-Garcia I, Stephens JM, Villarroya F. Oncostatin m impairs brown adipose tissue thermogenic function and the browning of subcutaneous white adipose tissue. Obesity (Silver Spring). 2017;25(1):85-93.

27. Sabench Pereferrer F, Domínguez-Adame Lanuza E, Ibarzabal A, et al; Sociedad Española de Cirugía de la Obesidad (SECO). Quality criteria in bariatric surgery: consensus review and recommendations of the Spanish Association of Surgeons and the Spanish Society of Bariatric Surgery. Cir Esp. 2017;95(1):4-16.

28. Piquer-Garcia I, Campderros L, Taxerås SD, et al. Data from: A Role for Oncostatin $\mathrm{M}$ in the Impairment of Glucose Homeostasis in Obesity. GitHub depository. Deposited 6 of September 2019. ProMED-mail website. https://github.com/ dsanchez79/Spplemental-Material.

29. Wabitsch M, Brenner RE, Melzner I, et al. Characterization of a human preadipocyte cell strain with high capacity for adipose differentiation. Int J Obes Relat Metab Disord. 2001;25(1):8-15.

30. Yeo CR, Agrawal M, Hoon S, et al. SGBS cells as a model of human adipocyte browning: a comprehensive comparative study 
with primary human white subcutaneous adipocytes. Sci Rep. 2017;7(1):4031.

31. Stephens JM, Bailey JL, Hang H, et al. Adipose tissue dysfunction occurs independently of obesity in adipocyte-specific oncostatin receptor knockout mice. Obesity (Silver Spring). 2018;26(9):1439-1447.

32. Elks CM, Zhao P, Grant RW, et al. Loss of Oncostatin M Signaling in Adipocytes Induces Insulin Resistance and Adipose Tissue Inflammation in Vivo. J Biol Chem. 2016;291(33):17066-17076.

33. Eckel N, Li Y, Kuxhaus O, Stefan N, Hu FB, Schulze MB. Transition from metabolic healthy to unhealthy phenotypes and association with cardiovascular disease risk across BMI categories in 90257 women (the Nurses' Health Study): 30 year follow-up from a prospective cohort study. Lancet Diabetes Endocrinol. 2018;6(9):714-724.

34. Eckel N, Meidtner K, Kalle-Uhlmann T, Stefan N, Schulze MB. Metabolically healthy obesity and cardiovascular events: a systematic review and meta-analysis. Eur J Prev Cardiol. 2016;23(9):956-966.

35. Phillips CM. Metabolically healthy obesity: definitions, determinants and clinical implications. Rev Endocr Metab Disord. 2013;14(3):219-227.

36. Karelis AD, Faraj M, Bastard JP, et al. The metabolically healthy but obese individual presents a favorable inflammation profile. $J$ Clin Endocrinol Metab. 2005;90(7):4145-4150.

37. Koster A, Stenholm S, Alley DE, et al; Health ABC Study. Body fat distribution and inflammation among obese older adults with and without metabolic syndrome. Obesity (Silver Spring). 2010;18(12):2354-2361.

38. Komori T, Tanaka M, Senba E, Miyajima A, Morikawa Y. Lack of oncostatin $M$ receptor $\beta$ leads to adipose tissue inflammation and insulin resistance by switching macrophage phenotype. J Biol Chem. 2013;288(30):21861-21875.

39. Komori T, Tanaka M, Senba E, Miyajima A, Morikawa Y. Deficiency of oncostatin $M$ receptor $\beta$ (OSMR $\beta$ ) exacerbates high-fat diet-induced obesity and related metabolic disorders in mice. J Biol Chem. 2014;289(20):13821-13837.

40. Komori T, Tanaka M, Furuta H, Akamizu T, Miyajima A, Morikawa $\mathrm{Y}$. Oncostatin $\mathrm{M}$ is a potential agent for the treatment of obesity and related metabolic disorders: a study in mice. Diabetologia. 2015;58(8):1868-1876.

41. Albiero M, Poncina N, Ciciliot S, et al. Bone Marrow Macrophages Contribute to Diabetic Stem Cell Mobilopathy by Producing Oncostatin M. Diabetes. 2015;64(8):2957-2968.

42. Elks CM, Stephens JM. Oncostatin m modulation of lipid storage. Biology (Basel). 2015;4(1):151-160.

43. Rega G, Kaun C, Weiss TW, et al. Inflammatory cytokines interleukin-6 and oncostatin $\mathrm{m}$ induce plasminogen activator inhibitor-1 in human adipose tissue. Circulation. 2005;111(15):1938-1945.

44. Catalan-Serra I, Brenna Ø. Immunotherapy in inflammatory bowel disease: novel and emerging treatments. Hum Vaccin Immunother. 2018;14(11):2597-2611. 